\title{
Dynamic Interaction System Design of Urban Landscape Information Based on PHP Technology
}

\author{
Wang Shun-Hui and Zou Jie \\ Institute of Arts and Technology, Harbin University of Science and Technology, \\ China \\ College of Foreign Languages, Harbin University of Science and Technology, \\ China \\ a.shunhuiwang@yahoo.com,b.en.landscape@gmail.com
}

\begin{abstract}
In the transitional period of Urbanization, the urban landscape and its aesthetical experience became one of research priorities. Design and develop professional interactive information system is one of the most important thing for urban landscape design. The research uses PHP as core technology, uses DW which is the integration of MySQL-related technologies as developing tools, to set up a integrated system platform based on Apache, MySQL and PHP. It is a dynamic interactive information system of urban landscape based on Web technologies. This article described the structure and functions of the system, explained design methods of the database and application. The construction and application of the system can integrate landscape information of the transitional period of Urbanization, such as item information, engineering information, education information and artistic information, offer a human-machine interactive communication platform based on P2P mode (point-to-point). It will become innovative power for the development of urban landscape industry.
\end{abstract}

Keywords: Dynamic interaction, PHP, MySQL, Urban landscape, Art Science and Technology

\section{Introduction}

Urban Landscape is a new industry which is very broad, comprehensive, and difficult to accurately define. City is a complicated organism; its main body is building. Building and space environment outside of the building, they called the urban landscape. Thus it can be seen that urban landscape has the characteristic of construction industry, at the same time, it has the characteristic of visual arts and behavioral psychology. It will be of great importance in the citizens' understanding of the city, field experience, and formation of homeland consciousness. Urban Landscape lets people living in it feel healthy, happy and comfortable. It has rich connotation of material life and spiritual life. [1]

Because of the importance of urban landscape, its informationization is urgently needed. Using network information technologies such as PHP, the research offered a development scheme of information system for urban landscape industry. The informatization of urban landscape industry will become an example of combination between urban landscape artistic design and technologies.

\subsection{The Development of PHP and Technical Characteristics}

PHP has been in use since 1995. A programmer of the United States whose name was Rasmus Lerdor, developed a Perl/CGI script that was used to take control of his own online data. At that time, it had only two basic functions: 1. Record the basic information 
of the visitor into log. 2. Page calculated and showed the access number. This script created a technological age, and Lerdorf called it PHP (Personal Home Page).

Later, the developers who like the function gathered together, developed new function which can turn input data of HTML Form to symbolic variable, export and share data to other system. In 1997, PHP2 was published, which was called PHP/FI at that time. In 1998, PHP3 had more than 50000 loyal users as soon as it was born. In 1999, data published by Netcraft(a company engaged in the Internet research and analysis) showed that the users of PHP was more than 1 million. At the beginning of 2000, PHP4 was published; data published by Netcraft showed that the user was more than 3.6 million. So far, PHP had become one of the most popular scripting languages in the world. According to the most conservative projection, PHP5 have more than 20 million users. PHP 5 developed Object-Oriented functionality, improved Zend Framework, has haven the functional characteristics as first-class programming language.

PHP has a significant advantage over HTML. It can contain HTML tags, script command and COM components. It has the functions of adding interactive content to the page, creating the HTML page as a Web application. The users of PHP don't need to use a particular browser; they can use ordinary browsers that can perform HTML code. All the PHP scripting language can be executed in the Web server, its source code will not be transmitted to the client browser, greatly reduce the risk that source program will be stolen, improve the security of the system.[2]Using PHP as the core technology is the choice of most websites, as shown in Figure 1.

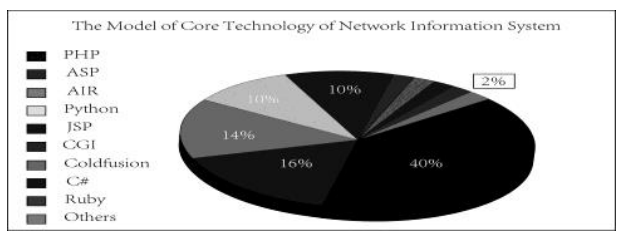

Figure 1. The Model of Core Technology of the Network Information System

PHP is script-embedded language; absorb the advantages of a variety of languages such as C, Perl, and JavaScript. The code is tight, grammatical structure is clear. The main technical features include data type, identifier, control statement, character string, POSIX, function, array, OOP, etc.

PHP is by far the most popular network system construction and web development language. From the speed, memory management, database collocation, portability and development costs, such as point of view, have comprehensive advantages. Many of the world's famous web sites using PHP core technology, most at the same time using Linux and MySQL database. As shown in table 1.

Table 1. Famous Website Using PHP Technology

\begin{tabular}{cccc}
\hline WebSite & Programming Language & OS & Database \\
\hline Facebook & PHP & Linux + Apache & MySQL \\
Yahoo & PHP & FreeBSD+Apache & MySQL \\
Baidu & PHP & Linux+Apache & MySQL \\
Wikipedia & PHP & Linux+Apache & MySQL \\
Taobao & PHP & Linux & Oracle \\
Sina & PHP & Linux+Apache & MySQL \\
BKOHTAKTE & PHP & Linux + Apache & MySQL \\
Tumblr & PHP & Linux + Apache & MySQL \\
\hline
\end{tabular}

\subsection{The Creation of System and DW Development Environment}

PHP can create development environment or run network system by the PHP technical support. Under the Windows system, it can install LLS or Apache web server. Under the Linux system, can use Apache as server. 
PHP can choose code editor widely. Under the Linux system, Bluefish can be chosen. Under the Windows system, DW (Dreamweaver), Notepad++, PDT, Zend Studio can be chosen. Each of the code editor above has advantages, here we chose more popular DW (Dreamweaver). DW integrates a variety of key technologies, such as AJAX, CSS, HTML, JavaScript, PHP, XML, and so on. It is called one-stop application program. DW is able to write and manage the code efficiently, highlight grammar, realize code completion. It can be used to develop the cutting edge of the network information system. [3]

To build the information system based on PHP, we have to install the Apache web server program and SQL server (the latter is recommended to use MySQL). The three software were being selected because their additional uses are mature in technology, and there is all low-cost open source software.

\section{The Construction and Whole Design Thinking of the Dynamic Interactive Information System Of Urban Landscape}

By the dynamic interactive information system of urban landscape, we can collect landscape information, discuss the urban construction regulations, and solicit suggestions for the construction of public area of the city. It can help us to promote the urban grade around the city, and improve the human settlements environment. Through the browser on the client devices of computer or mobile phone, the citizens can visit the system; realize the interaction with urban management, policy making, development parties, and the researchers of urban landscape. The system has functions of user registration, logging, and research online. It makes it possible that different user's access to the same address. System can realize the user register, login, online research, file downloads, e-paper, and even for different landscape project related functions such as Internet voting. According to the requirements of the system, different user's access to the same address show different page also will be one of the basic functions of this system.

\subsection{Operating Principle of Dynamic Pages}

In order to achieve the above functions, must have a dynamic page technology support. Dynamic pages are a kind of web technology against static web pages. Static web pages as soon as created by html code, contents of the pages and show effects cannot be changed. But contents of dynamic pages can be changed by time, environment, and the results of the database operation. It is not directly related to all kinds of the display of dynamic arrows, the dynamic effects on the vision like rolling captions. These just are the representations. No matter whether have dynamic effect, as long as the pages created by dynamic web technology, all can be called dynamic pages. [4]

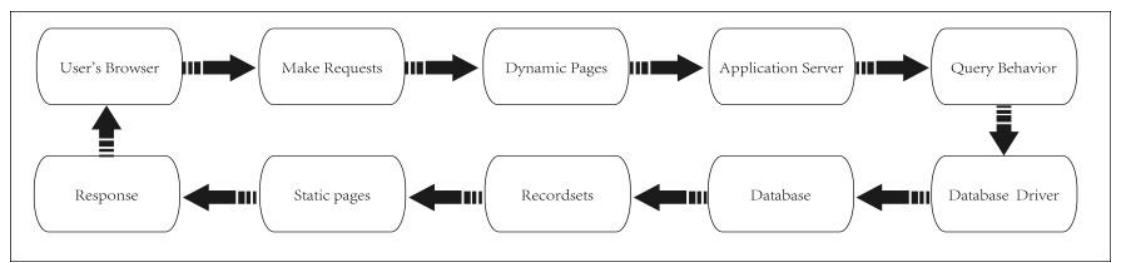

Figure 2. Working Principle of Urban Landscape Dynamic Interaction Information System

Dynamic pages need to have technical support database as the background. The coordination between downstage page and back-end database realized dynamic update of page. Its working principle is as follows: client-browser sends out requests by opening the dynamic page, after the server received the request signal, the web page will be sent to the server. The network server will validate the legitimacy of the request, if the request has 
been verified, will be sent to the database driver. The database driver make queries to the back-end database send back record set to database driver. Then the record set will be sent to the server. Finally, the server inserts data to a web page. Static web pages will be turned from dynamic pages, and then be sent to client-browser. Client-browser will explain and transform HTML language; the results can be seen at user's display.

\subsection{The Whole Design Thinking and Construction}

The whole design thinking includes the way of content presentation, the way of browsing, the way of dynamic interaction, the system feature set, visual effect and User's interaction experience. We must consider how to meet the demands of users who need a variety of urban landscape information, in order to construct and use the system better.

After the functional orientation has been done, we have to plan the construction of the system. Because network information system uses tree structure, the division of level and connection between each part are important. The construction of the interactive information system of urban landscape is shown in Figure 3.

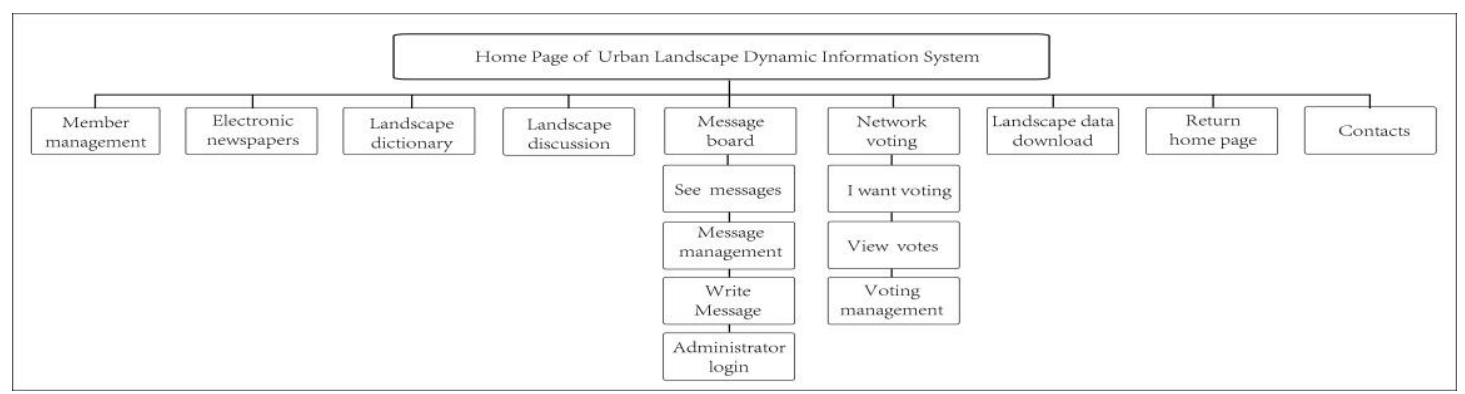

Figure 3. Model of Information Categories

Based on above construction, according to main content, the leading page, the index page, and inside pages will be planned. According to schedule t designed, the work will be divided to project managers, planners, visual effect engineer and programming engineer. In their joint efforts, the development of the system will be done.

\subsection{Development and Design of the Interactive Information System of Urban LandsCape}

Database is important backend technology for the interactive information system. Database is made of a number of data tables directly related to the system, and a number of databases crease the relational database. Data table is made up of several variable fields. The type of field is rich, including auto number, remark, text, number, currency, date and time, OLE object(object linking embedded, referring to the picture and sound, etc), hyperlink, and soon. SQL (Structured query language) is structured query language; its effect is to query the database records, process data through commands. It also can realize remote data processing by programming language such as PHP. SQL was first postulated by E.F.Codd in 1970. After several years of development, it was developed to standard language of commercial database by IBM. In 1986, ANSI (American national standards institute) takes SQL as national standard. SQL command type includes two types: DDL (Date definiton language) and DML(Date Manipulation language). The main commands include CREATE, ALTER, DROP, SELECT, ORDER BY, GROUP BY, HAVING, DISTINCT, LIMIT, INSERT, UPDATE, DELETE, etc. The interactive information system of urban landscape uses MySQL as database server. [5] Its ER Model to Relations is shown as Figure 4. 


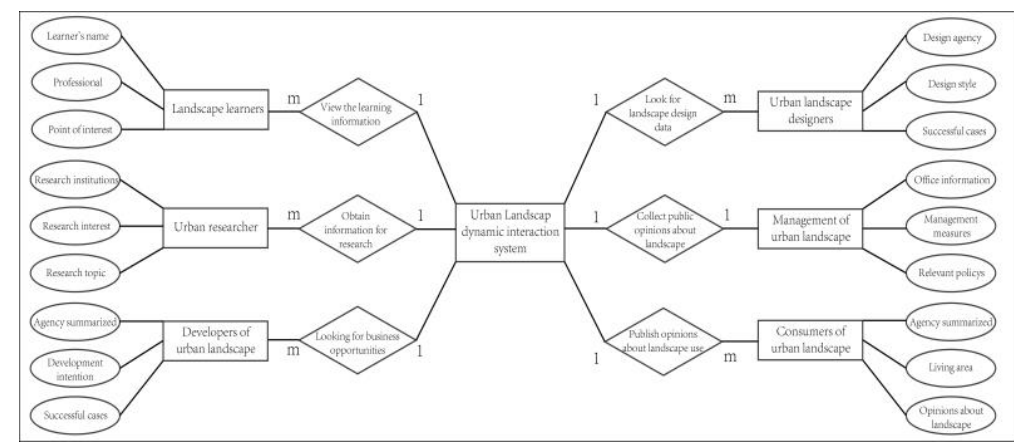

Figure 4. E-R Model of Urban Landscape Information System

\section{Application of Dynamic Page Technology in the Dynamic Interactive Information System}

\subsection{Design of the Dynamic Interactive Information System of Urban Landscape by PHP-In The Case Of Member Login Module}

PHP Technology And Dynamic Page Structure: PHP is a kind of HTML document that can be implemented on the server side, execute script by WEB server, create the HTML language, then applied to the front, file name is "*.php". The basic mechanism includes 4 parts: 1. HTML-tags. All the HTML-tags can be used in PHP. 2. PHP verbal order is located in the $<$ ? PHP $>$ tag. 3. Include function. Call the other code on the server side. 4. Scripting language. C,Java,Perl, all can be used by PHP, Enhance the function of programming. [6]

3.1.2. Design of System Login Modules for the System: System login modules are embodied in effective management of EV members. When users logged into the system remotely, they firstly contact the system login module. This module is important way for the system to identify users, is basis for system to lead the users into different pages. The users register information to the database through registration page, the system read and verify the user's information from database. In the process, the key is the collocation between downstage page and background database. The system login modules of the system are shown in Figure 5.The fields of the data table is shown in Table 2.

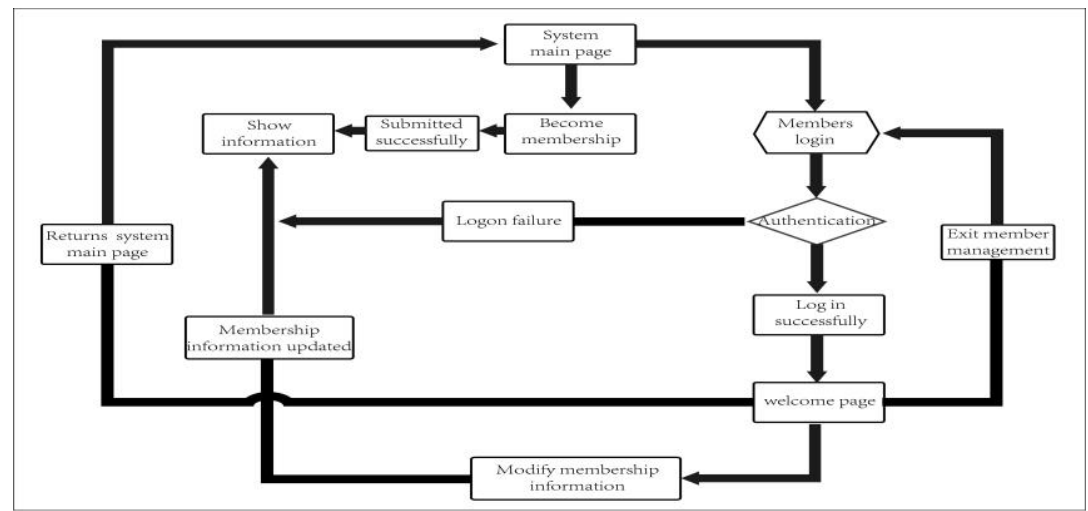

Figure 5. Members Login Module Running Model of Urban Landscape Information System 
Table 2. Field Design of Members Login Module

\begin{tabular}{|cccc|}
\hline Field & Type & Length & Instructions \\
\hline Landscape_id & Integer & 11 & Save the membership record number, automatically generated by the database \\
Landscape_no & String & 14 & Save the member account, finite length is 14 characters \\
Landscape_pwd & String & 10 & Save the member password, finite length for 10 characters \\
Landscape_name & String & 40 & Save the member name, finite length is 40 characters \\
Landscape_sex & Integer & 1 & Save the gender \\
Landscape_birth & String & 10 & Save the birthday, user input 8 code date format \\
Landscape_add & String & 200 & Save the address, finite length is 200 characters \\
Landscape_tel & String & 14 & Save the phone, finite length is 14 characters \\
Landscape_email & String & 100 & Save the email,finite length is 100 characters \\
\hline
\end{tabular}

\subsection{Application of Dynamic Web Page in the Dynamic Interactive Information System of Urban Landscape}

The grammar of PHP is very near to $\mathrm{C}$ language,programming style is near to $\mathrm{C}++$, put the code in the descriptor <?php PHP ? >. In the process of building foreground and background, we used MySQL. In the PHP sentences inserted in HTML pages, specify the alternative paths such as the name of the database server, account number and password, put in the sentence of connecting to the database, then we can write SQL query statement. The query results in the form of a record set, display on the html page by echo or printf function, then return to the client browser. The system uses MySQL database server, the development process is as follows [7].

The database is installed on the computer called"institute of art and technology", set up a user account as "landscape group". In the database "urban_landscape", there is a table called"information system".There are three fields: Developer, Planners, and Designer. Users can not see that PHP is performed on the server side. The result will return to the user's browser.

$\langle$ html $>$

$<$ body $>$

$<$ ?php

$\$ \mathrm{db}=$ mysql_connect("institute of art and technology","landscape_group");//mysql_connect（） specify a user name (institute of art and technology) connect to the databaseon the specified computer

(landscape_group) , save the structure resulting from the connection to variable $\$ \mathrm{db}$

mysql_select_db(“urban_landscape”,\$db);//mysql_select_db ( ) account data to operate is (urban_landscape)

\$result=mysql_query(“SELECT*FROM information_system”\$db);//mysql_query（） make use of the connection according to the results is $\$ \mathrm{db}$, the function to submit SQL query for MYSQL.recordset returned is stored to\$result.

printf(“" $<$ table border $=1>\backslash \mathrm{n}>$ ");

printf( “ $<\mathrm{tr}><\mathrm{td}>$ Landscape

developers $</ \mathrm{td}\rangle\langle\mathrm{td}\rangle$ Landscape designers $/ \mathrm{td}\rangle\langle/ \mathrm{tr}\rangle \mid \mathrm{n}$ ");

planners $</ \mathrm{td}><\mathrm{td}>$ Landscape

while(\$myrow=mysql_fetch_row(\$res-ult)); //mysql_fetch_rowexplain\$result,it return to the first array, use data subscript to access records in the field.the subscript of first field is 0 , the subscript of second field is 1 , and so on.

\{printf(“ $<$ tr $><$ td $>\% \mathrm{~s}</ \mathrm{td}><\mathrm{td}>\% \mathrm{~s}</ \mathrm{td}><\mathrm{td}>\% \mathrm{~s}</ \mathrm{td}></$ tr $>$ In", Smyrow[1]

\$myrow[2],\$myrow=[3]);//shown the table of city landscape project information of three fields related 


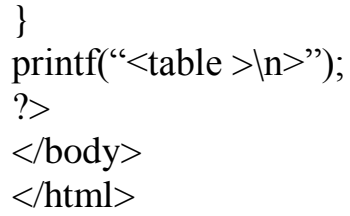

The interaction and dynamic is the key of the system. We must distinguish the identities of users, verify them, ensure the encryption process in transmitting procedure. In the case of user authentication module, crease submit checkuser.php fill, complete inspection operation of user name. The key code is as follows.

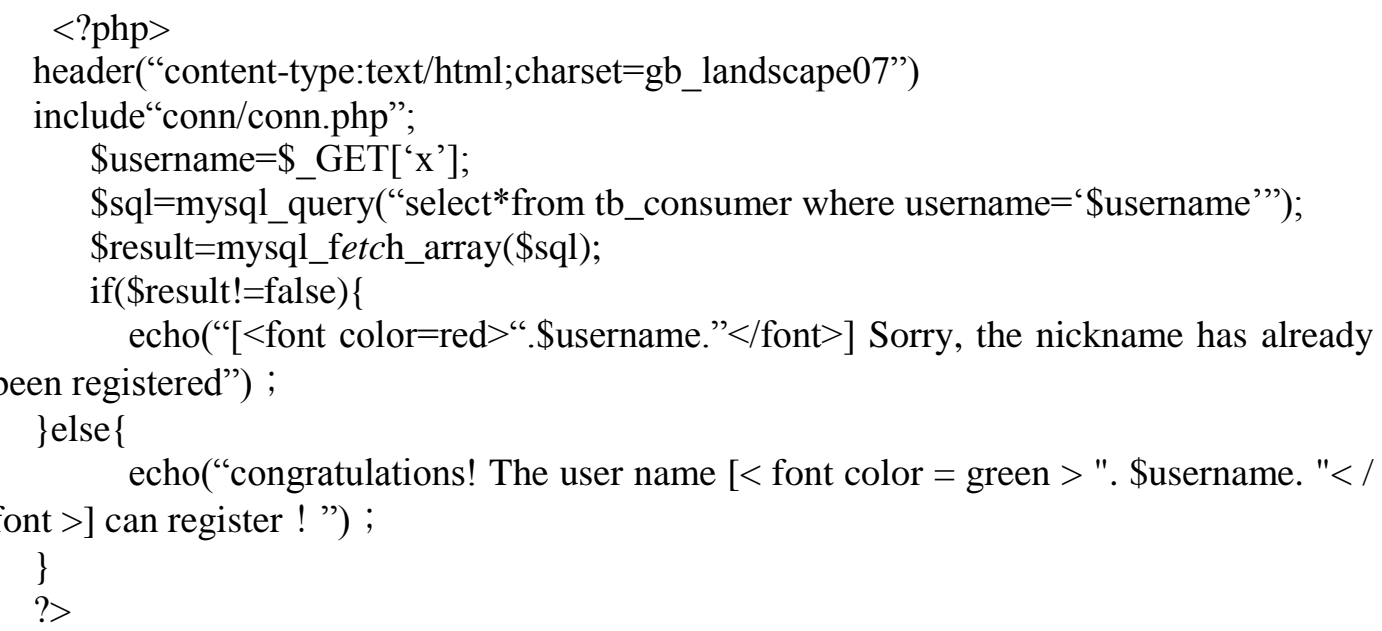

\subsection{Test and Release}

There are many forms to choose, including setting Host WEB, Dedicdinedd Hosting, free space, direct external connection, etc. All of them need upload the system page to the remote service by the way of uploading files.The upload ways include browser-based uploads and FTPbased uploads. The former focuses on the free space to upload; there are many restrictions in file size. The latter is convenient to use, but is generally for paying customers.

After multiple tests, the system's main process is: 1. Apply for website. 2. Apply for virtual space. Both of them have free and paid ways. Free ones are affiliate website, is not suitable for the development of this system. The paid ones are more independent, and stable and reliable. Many of ISP (internet service provider) companies provide virtual hosting service to let us choose. The release needs to set internet and firewall, then use FTP upload system, test the local test file for the system in the server.

\section{Conclusion}

The interactive information system of urban landscape is important part of strategies of new urban landscape. It has the vital practical significance in the transitional period of urbanization. The research used technologies such as PHP, MySQL, Apache, to build DW development environment, and crease a dynamic interactive information system including urban landscape information, project bidding information, news release, interactive information, and user experience information. The system has the characteristic of urban landscape industry. Considering the requirements of designers, planners, managers and users, we used modern information technologies. It realized the informatization by high integration of arts and information technologies. 


\section{Acknowledgements}

This research supported by Philosophy and Social Sciences Planning project of Heilongiiang Province (Project No.14E062).The author is director of Arts and Technology Institute, Harbin University of Science and Technology, research in the field cross of arts, Technology and social sciences.

\section{Reference}

[1] J. Leskovec, K. J. Lang, D. Anirban, et. al., "Statistical Properties of Community Structure in Large Social and Information Networks", Proceeding of the17th international conference on World Wide Web, vol.27, (2008), pp.18-22.

[2] W. Haiying, "The Research and Implement of Moderate Enterprise-Online Self-Help Platform", "Natural Science Journal of Harbin Normal University, vol. 2, no. 18, (2002).

[3] B. Ji, X. Luo, X. Peng, Y. Wu and H. Xu, "Numerical analysis of cavitation evolution and excited pressure fluctuation around a propeller in non-uniform wake", International Journal of Multiphase Flow (2012).

[4] E. Mohamad, "Electroic design for portable electrical capacitance sensor", a multiphase follow measurement, 4th Int, con, on Mecaha, (2011).

[5] X. Jiazhong and Y. Bo, "Research on Robot Off-line Programming System for High-pressure Alter-jet Cutting", Journal of Harbin University of Science and Technology, vol. 14, no. 2, (2009).

[6] P. L. Rizet, "Frequency behavior and speed control of piezom otors", Sensors and Actuators, vol. 1 , no. 80, (2000).

[7] L. Ullman, "PHP and MySQL for Dynamic Web Sites", Visual QuickPro Guide (4th Edition).M. Peachpit Press, 4 edition, vol. 9, no. 23, (2011). 